\title{
Nonequilibrium effects in the Casimir force between two similar metallic plates kept at different temperatures
}

\author{
G.-L. Ingold $\odot,{ }^{1}$ G. L. Klimchitskaya $\odot,{ }^{2,3}$ and V. M. Mostepanenko $\odot^{2,3,4}$ \\ ${ }^{1}$ Universität Augsburg, Institut für Physik, 86135 Augsburg, Germany \\ ${ }^{2}$ Central Astronomical Observatory at Pulkovo of the Russian Academy of Sciences, Saint Petersburg 196140, Russia \\ ${ }^{3}$ Institute of Physics, Nanotechnology and Telecommunications, Peter the Great Saint Petersburg Polytechnic University, \\ Saint Petersburg 195251, Russia \\ ${ }^{4}$ Kazan Federal University, Kazan 420008, Russia
}

(Received 17 December 2019; accepted 18 February 2020; published 11 March 2020)

\begin{abstract}
We study the Casimir pressure between two similar plates of finite thickness kept at different temperatures in the case when the dielectric permittivity of the plates depends on temperature. It is suggested to consider the dielectric permittivity at two different temperatures as the permittivities of two dissimilar bodies, thus, allowing to apply the theory of Casimir forces out of thermal equilibrium developed earlier in the literature. Following this approach, we show that, in addition to the equilibrium contribution to the nonequilibrium Casimir pressure, a proper nonequilibrium contribution arises for temperature-dependent dielectric permittivities. Furthermore, the equilibrium contribution in this case does not equal the mean of the equilibrium Casimir pressures at the temperatures of the plates. As an application, the total nonequilibrium Casimir pressure between two gold plates and between two titanium plates is calculated as a function of the plate thickness and their separation using the Drude and the plasma models. For plate separations ranging from 0.5 to $2 \mu \mathrm{m}$, the relative difference between the theoretical predictions for these two models reaches $39 \%$. The proper nonequilibrium term may be as large as $4 \%$ of the magnitude of the total nonequilibrium pressure.
\end{abstract}

DOI: 10.1103/PhysRevA.101.032506

\section{INTRODUCTION}

Electromagnetic fluctuations and fluctuation-induced phenomena, such as the Casimir and Casimir-Polder forces, are of widespread relevance and have gained increasing interest due to advances in experimental techniques allowing for the fabrication of various microstructures and their manipulation on the nanometer scale [1]. Different elements of a microstructure may be kept at different temperatures, i.e., out of thermal equilibrium. This raises the question of whether the standard Lifshitz theory describing the Casimir and Casimir-Polder forces $[2,3]$ can be generalized to account for nonequilibrium conditions.

The extension of the Lifshitz formula to the case of two semispaces made of absorbing materials at different temperatures was obtained in Ref. [4] in the framework of fluctuational electrodynamics. For the special situation where both semispaces are filled with the same material, it was shown that the nonequilibrium Casimir pressure is given by the mean of the equilibrium Casimir pressures at the temperatures of the two semispaces [4]. The Casimir-Polder force between an atom and a plate whose temperature is different from that of the environment was considered in Refs. [5,6]. We note also Ref. [7] where a theory of electromagnetic fluctuations has been used to compute the radiative heat transfer between two parallel metallic surfaces kept at different temperatures.

The general theory of Casimir and Casimir-Polder interactions in nonequilibrium situations was developed in Refs. [8,9]. In the framework of this theory, the Casimir pressure out of thermal equilibrium was presented as a sum of three contributions: the mean of two equilibrium pressures at respective temperatures, a proper nonequilibrium contribution, and a term which is independent of the separation between the plates. For two semispaces made of identical materials, it was confirmed that the Casimir pressure out of thermal equilibrium reduces to the mean of two equilibrium pressures. This is also true for two identical bodies of arbitrary shape placed symmetrically with respect to a plane [9]. Later, the theory of the Casimir force out of equilibrium was generalized to two or more arbitrarily shaped bodies consisting of dissimilar materials [10-17]. In Ref. [18], the possibility of a nonequilibrium repulsive force was proposed. Furthermore, the nonequilibrium Casimir force was considered in connection with the effect of radiative heat transfer, noncontact friction [19-22], and actuation of microdevices [23].

It is well known that the standard Lifshitz theory at thermal equilibrium faces problems in describing the Casimir free energy and the pressure between two metallic plates (see reviews [24,25] and the monograph [26]). Specifically, it turns out that the results for the Casimir interaction obtained by all high-precision experiments at separations below $1.1 \mu \mathrm{m}$ are in conflict with theoretical predictions taking into account the relaxation properties of conduction electrons [27-38]. If these relaxation properties are disregarded in computations, the Lifshitz theory is brought to a very good agreement with the measurement data [27-38]. Even going beyond the usually employed proximity force approximation by means of numerically exact calculations does not help to resolve this 
discrepancy $[39,40]$. How the experimental observations can be brought into agreement with the presence of relaxation processes for the conduction electrons remains a puzzle [41].

An investigation of the Casimir force out of thermal equilibrium may shed more light on the Casimir puzzle and point the way for its resolution. This line of attack was already used in Refs. [42,43] where the nonequilibrium Casimir force between metallic test bodies was calculated with and without taking into account relaxation properties of the conduction electrons, and different possibilities to measure it were proposed. Arguing that corrections due to the temperature dependence of the dielectric permittivity would be small compared to the effect of a temperature difference between the plates, the dielectric permittivity was assumed to be temperature independent.

In the present paper, we consider the Casimir pressure between two similar metallic plates of finite thickness kept at different temperatures and taking into account the temperature dependence of their dielectric permittivity. Although the general theory of Refs. [8,9] does not explicitly account for such a temperature dependence, it allows for different materials and, therefore, is still applicable in our case. Then, one should consider the plate material at two different temperatures as two dissimilar materials with different dielectric permittivities depending only on frequency.

Following this approach, we demonstrate that a dependence of the dielectric permittivity on temperature leads not only to quantitative, but also to important qualitative effects. Specifically, we show that, for two similar metallic plates, the nonequilibrium Casimir pressure does not reduce to the mean of equilibrium pressures taken at the two different temperatures of the plates as would be the case for bodies with temperature-independent dielectric permittivity $[4,8,9]$. Not only is this equilibrium contribution modified, but also an additional proper nonequilibrium term contributes to the total pressure.

As an application of the approach just outlined, we have performed numerical computations of the Casimir pressure between two similar plates of finite thickness made of either $\mathrm{Au}$ or Ti kept at different temperatures. The distance between the plates varies from 0.5 to $2 \mu \mathrm{m}$ where we can restrict our consideration to either the Drude or the plasma model [26]. The plasma frequency describing the high-frequency transparency of metals appears in both models and can be taken as independent of temperature. In addition, the Drude model includes relaxation properties of the conduction electrons where the temperature dependence needs to be taken into account.

We have computed the magnitudes of the nonequilibrium Casimir pressure as a function of separation for different plate thicknesses using either the Drude or the plasma model. The corresponding results differ significantly and can be discriminated experimentally. Furthermore, we determined the relative difference between the standard and the modified equilibrium contributions to the nonequilibrium Casimir pressure. The magnitude of this quantity was found to be less than $1 \%$. We have also computed the values of the proper nonequilibrium contribution to the nonequilibrium Casimir pressure at different separations and plate thicknesses. It is shown that this contribution can reach $4 \%$ of the magnitude of the total nonequilibrium pressure.

The paper is organized as follows. In Sec. II, the general formalism is presented which allows to calculate the nonequilibrium Casimir pressure between two similar plates with temperature-dependent dielectric permittivity. Section III contains our computational results for two similar plates made either of gold or of titanium. In Sec. IV, we will present our conclusions and a discussion.

\section{GENERAL FORMALISM FOR SIMILAR METALLIC PLATES OUT OF THERMAL EQUILIBRIUM}

We consider the configuration of two parallel metallic plates of thickness $d$ in vacuum consisting of the same material and separated by a distance $a$ as depicted in Fig. 1. The upper plate is kept at the temperature $T_{1}$ assumed to equal the temperature of the environment whereas the lower plate has a different temperature $T_{2}$. The plate material is characterized by a dielectric permittivity $\varepsilon(\omega, T)$ which, in addition to its frequency dependence, may, in general, also vary with temperature. In this case, one can consider the two plates as effectively consisting of different materials with dielectric permittivities $\varepsilon^{(1)}(\omega)=\varepsilon\left(\omega, T_{1}\right)$ and $\varepsilon^{(2)}(\omega)=\varepsilon\left(\omega, T_{2}\right)$, respectively.

Then, using the theory developed in Ref. [9], the nonequilibrium Casimir pressure on the lower plate can be expressed as the sum of two contributions,

$$
P_{\text {neq }}\left(a, T_{1}, T_{2}\right)=\widetilde{P}_{\text {eq }}\left(a, T_{1}, T_{2}\right)+\Delta P_{\text {neq }}\left(a, T_{1}, T_{2}\right) .
$$

Here, the quantity $\widetilde{P}_{\text {eq }}$ is a modification of the standard equilibrium contribution of Refs. $[4,9,18]$,

$$
\bar{P}_{\mathrm{eq}}\left(a, T_{1}, T_{2}\right)=\frac{1}{2}\left[P_{\mathrm{eq}}\left(a, T_{1}\right)+P_{\mathrm{eq}}\left(a, T_{2}\right)\right]
$$

equal to the mean of Casimir pressures calculated for the cases when both plates are maintained either at the temperature $T_{1}$ or $T_{2}$. The quantity $\Delta P_{\text {neq }}$ is the proper nonequilibrium contribution to $P_{\text {neq }}$ which vanishes for two similar plates possessing a temperature-independent dielectric permittivity.

The explicit expression for $\widetilde{P}_{\mathrm{eq}}$ is given by

$$
\begin{aligned}
\widetilde{P}_{\mathrm{eq}}\left(a, T_{1}, T_{2}\right)= & -\frac{k_{B}}{2 \pi}\left[T_{1} \sum_{l=0}^{\infty} \int_{0}^{\infty} q_{l}^{(1)} k_{\perp} d k_{\perp} \sum_{\alpha} \frac{R_{\alpha}\left(i \xi_{l}^{(1)}, k_{\perp}, T_{1}\right) R_{\alpha}\left(i \xi_{l}^{(1)}, k_{\perp}, T_{2}\right)}{e^{2 q_{l}^{(1)} a}-R_{\alpha}\left(i \xi_{l}^{(1)}, k_{\perp}, T_{1}\right) R_{\alpha}\left(i \xi_{l}^{(1)}, k_{\perp}, T_{2}\right)}\right. \\
& \left.+T_{2} \sum_{l=0}^{\infty} \int_{0}^{\infty} q_{l}^{(2)} k_{\perp} d k_{\perp} \sum_{\alpha} \frac{R_{\alpha}\left(i \xi_{l}^{(2)}, k_{\perp}, T_{1}\right) R_{\alpha}\left(i \xi_{l}^{(2)}, k_{\perp}, T_{2}\right)}{e^{2 q_{l}^{(2)} a}-R_{\alpha}\left(i \xi_{l}^{(2)}, k_{\perp}, T_{1}\right) R_{\alpha}\left(i \xi_{l}^{(2)}, k_{\perp}, T_{2}\right)}\right] .
\end{aligned}
$$




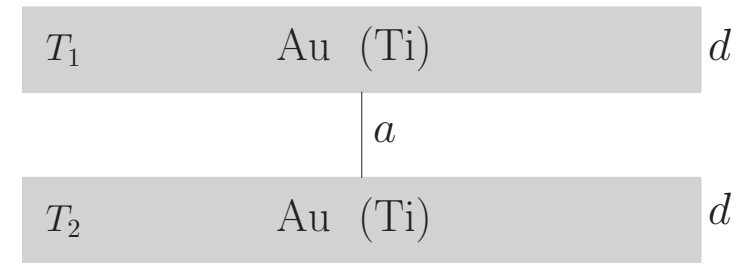

FIG. 1. The configuration of two parallel plates of thickness $d$ which are kept at different temperatures $T_{1}$ (as in the environment) and $T_{2}$.

Here, $k_{B}$ is the Boltzmann constant, $k_{\perp}$ is the magnitude of the wave vector projected onto the plane defined by the plate surface, and the Matsubara frequencies $\xi_{l}^{(n)}$ with $n=1,2$ are defined as $\xi_{l}^{(n)}=2 \pi k_{B} T_{n} l / \hbar, l=0,1,2, \ldots$. The prime on the summation in $l$ divides the term with $l=0$ by 2 , and $q_{l}^{(n)}$ is defined as

$$
q_{l}^{(n)}=\sqrt{k_{\perp}^{2}+\frac{\xi_{l}^{(n)^{2}}}{c^{2}}} .
$$

The sums in $\alpha$ are over the two polarizations of electromagnetic waves, transverse magnetic $(\alpha=\mathrm{TM})$ and transverse electric ( $\alpha=$ TE). The reflection coefficients $R_{\alpha}$ on the plate of finite thickness $d$ are given by [26]

$$
R_{\alpha}\left(i \xi_{l}^{(n)}, k_{\perp}, T_{m}\right)=\frac{r_{\alpha}\left(i \xi_{l}^{(n)}, k_{\perp}, T_{m}\right)\left(1-e^{-2 d v_{l}^{(n, m)}}\right)}{1-r_{\alpha}^{2}\left(i \xi_{l}^{(n)}, k_{\perp}, T_{m}\right) e^{-2 d v_{l}^{(n, m)}}},
$$

where $m=1,2$ denotes the plates and the Fresnel coefficients for the reflection at the surface of a semispace depend on the dielectric permittivity $\varepsilon(\omega, T)$ as

$$
\begin{aligned}
& r_{\mathrm{TM}}\left(i \xi_{l}^{(n)}, k_{\perp}, T_{m}\right)=\frac{\varepsilon\left(i \xi_{l}^{(n)}, T_{m}\right) q_{l}^{(n)}-v_{l}^{(n, m)}}{\varepsilon\left(i \xi_{l}^{(n)}, T_{m}\right) q_{l}^{(n)}+v_{l}^{(n, m)}}, \\
& r_{\mathrm{TE}}\left(i \xi_{l}^{(n)}, k_{\perp}, T_{m}\right)=\frac{q_{l}^{(n)}-v_{l}^{(n, m)}}{q_{l}^{(n)}+v_{l}^{(n, m)}},
\end{aligned}
$$

with

$$
v_{l}^{(n, m)}=\sqrt{k_{\perp}^{2}+\varepsilon\left(i \xi_{l}^{(n)}, T_{m}\right) \frac{\xi_{l}^{(n)^{2}}}{c^{2}}} .
$$

The expression (3) is nothing else than the quantity $P_{\text {eq }}$ of Refs. $[4,9,18]$ written for two plates made of materials with different reflection coefficients,

$$
\begin{aligned}
& R_{\alpha}^{(1)}\left(i \xi_{l}^{(n)}, k_{\perp}\right)=R_{\alpha}\left(i \xi_{l}^{(n)}, k_{\perp}, T_{1}\right), \\
& R_{\alpha}^{(2)}\left(i \xi_{l}^{(n)}, k_{\perp}\right)=R_{\alpha}\left(i \xi_{l}^{(n)}, k_{\perp}, T_{2}\right) .
\end{aligned}
$$

It should be pointed out, however, that (3) reduces to the mean (2) of two equilibrium contributions at temperatures $T_{1}$ and $T_{2}$ only when the dielectric permittivities are temperature independent.

Following the same approach, the proper nonequilibrium contribution to the nonequilibrium Casimir pressure (1) can be written as follows [9] (see also Ref. [18]):

$$
\begin{aligned}
\Delta P_{\text {neq }}\left(a, T_{1}, T_{2}\right)= & \frac{\hbar}{4 \pi^{2}} \int_{0}^{\infty} d \omega\left[\Theta\left(\omega, T_{1}\right)-\Theta\left(\omega, T_{2}\right)\right] \int_{0}^{\omega / c} d k_{\perp} k_{\perp} p \sum_{\alpha} \frac{\left|R_{\alpha}\left(\omega, k_{\perp}, T_{2}\right)\right|^{2}-\left|R_{\alpha}\left(\omega, k_{\perp}, T_{1}\right)\right|^{2}}{\left|D_{\alpha}\left(\omega, k_{\perp}, T_{1}, T_{2}\right)\right|^{2}} \\
& -\frac{\hbar}{2 \pi^{2}} \int_{0}^{\infty} d \omega\left[\Theta\left(\omega, T_{1}\right)-\Theta\left(\omega, T_{2}\right)\right] \int_{\omega / c}^{\infty} d k_{\perp} k_{\perp} \operatorname{Im} p e^{-2 a \operatorname{Im} p} \\
& \times \sum_{\alpha} \frac{\operatorname{Im} R_{\alpha}\left(\omega, k_{\perp}, T_{1}\right) \operatorname{Re} R_{\alpha}\left(\omega, k_{\perp}, T_{2}\right)-\operatorname{Re} R_{\alpha}\left(\omega, k_{\perp}, T_{1}\right) \operatorname{Im} R_{\alpha}\left(\omega, k_{\perp}, T_{2}\right)}{\left|D_{\alpha}\left(\omega, k_{\perp}, T_{1}, T_{2}\right)\right|^{2}}
\end{aligned}
$$

where we have introduced the thermal photon population,

$$
\Theta\left(\omega, T_{m}\right)=\frac{1}{\exp \left(\frac{\hbar \omega}{k_{B} T_{m}}\right)-1},
$$

the component of the wave vector perpendicular to the plate surfaces,

$$
p=\sqrt{\frac{\omega^{2}}{c^{2}}-k_{\perp}^{2}},
$$

and

$$
D_{\alpha}\left(\omega, k_{\perp}, T_{1}, T_{2}\right)=1-R_{\alpha}\left(\omega, k_{\perp}, T_{1}\right) R_{\alpha}\left(\omega, k_{\perp}, T_{2}\right) e^{2 i p a} .
$$

The reflection coefficients for a plate of thickness $d$ appearing in (9) as a function of real frequencies [44],

$$
R_{\alpha}\left(\omega, k_{\perp}, T_{m}\right)=\frac{r_{\alpha}\left(\omega, k_{\perp}, T_{m}\right)\left(1-e^{2 i d u^{(m)}}\right)}{1-r_{\alpha}^{2}\left(\omega, k_{\perp}, T_{m}\right) e^{2 i d u^{(m)}}},
$$

are expressed through the Fresnel coefficients,

$$
\begin{aligned}
r_{\mathrm{TM}}\left(\omega, k_{\perp}, T_{m}\right) & =\frac{\varepsilon\left(\omega, T_{m}\right) p-u^{(m)}}{\varepsilon\left(\omega, T_{m}\right) p+u^{(m)}}, \\
r_{\mathrm{TE}}\left(\omega, k_{\perp}, T_{m}\right) & =\frac{p-u^{(m)}}{p+u^{(m)}},
\end{aligned}
$$

where

$$
u^{(m)}=\sqrt{\varepsilon\left(\omega, T_{m}\right) \frac{\omega^{2}}{c^{2}}-k_{\perp}^{2}} .
$$

Like in the case of the equilibrium contribution discussed above, Eq. (9) is obtained from the respective result of Ref. [9] for two dissimilar plates by putting

$$
\begin{aligned}
& R_{\alpha}^{(1)}\left(\omega, k_{\perp}\right)=R_{\alpha}\left(\omega, k_{\perp}, T_{1}\right), \\
& R_{\alpha}^{(2)}\left(\omega, k_{\perp}\right)=R_{\alpha}\left(\omega, k_{\perp}, T_{2}\right) .
\end{aligned}
$$


If the dielectric permittivity is temperature independent, one obtains $R_{\alpha}^{(1)}=R_{\alpha}^{(2)}$ for two similar plates, and Eq. (9) leads to $\Delta P_{\text {neq }}=0[4,9,18]$. This is not the case, however, for two similar plates described by a temperature-dependent dielectric permittivity.

As was mentioned above, the nonequilibrium Casimir pressure (1) acts on the lower plate which is kept at the temperature $T_{2}$ different from the temperature of the environment. Then, the pressure on the upper plate kept at the temperature of the environment $T_{1}$ contains an additional contribution from the environmental blackbody radiation pressure [18,43],

$$
\widehat{P}_{\text {neq }}\left(a, T_{1}, T_{2}\right)=P_{\text {neq }}\left(a, T_{1}, T_{2}\right)+\frac{2 \sigma}{3 c}\left(T_{2}^{4}-T_{1}^{4}\right),
$$

where $\sigma$ is the Stefan-Boltzmann constant and $P_{\text {neq }}$ is defined through Eqs. (1), (3), and (9).

In the next section, we will make use of the formalism just presented in order to numerically determine the nonequilibrium Casimir pressure and its different contributions for two parallel metallic plates of finite thickness.

\section{COMPUTATIONAL RESULTS FOR GOLD AND TITANIUM PLATES}

To demonstrate physical consequences of the formalism presented in Sec. II, we consider two similar parallel metallic plates kept at temperatures of $T_{1}=300$ and $T_{2}=500 \mathrm{~K}$ and separated by distances $a \geqslant 0.5 \mu \mathrm{m}$. At such separations, the Casimir pressure is largely determined by the contribution of conduction electrons to the dielectric permittivity of a metal whereas the contribution of core electrons results only in minor corrections [26]. The chosen temperatures do not appear as unrealistic in view of existing experiments [45].

The most frequently used and well-confirmed dielectric permittivity describing the conduction electrons in metals including their relaxation properties is given by the Drude model,

$$
\varepsilon_{D}\left(\omega, T_{m}\right)=1-\frac{\omega_{p}^{2}}{\omega\left[\omega+i \gamma\left(T_{m}\right)\right]},
$$

where $\omega_{p}$ is the plasma frequency. This dielectric permittivity is temperature dependent through the temperature dependence of the relaxation parameter $\gamma\left(T_{m}\right)$. Here, $T_{m}$ is the temperature of a metallic plate in local thermal equilibrium [9] ( $T_{1}$ and $T_{2}$ for upper and lower plates, respectively). At the purely imaginary Matsubara frequencies $i \xi_{l}^{(n)}$, the permittivity (18) takes the form

$$
\varepsilon_{D}\left(i \xi_{l}^{(n)}, T_{m}\right)=1+\frac{\omega_{p}^{2}}{\xi_{l}^{(n)}\left[\xi_{l}^{(n)}+\gamma\left(T_{m}\right)\right]} .
$$

As discussed in Sec. I, theoretical predictions of the Lifshitz theory using the Drude model (or the optical data of metals extrapolated by means of the Drude model down to zero frequency) are excluded by high-precision measurements of the Casimir force. For reasons unknown to us, the measurement data are in agreement with the theoretical predictions disregarding the relaxation properties of conduction electrons.
TABLE I. Plasma frequency $\omega_{p}$ and relaxation parameter $\gamma$ at two different temperatures for gold and titanium.

\begin{tabular}{lccc}
\hline \hline Metal & $\hbar \omega_{p}(\mathrm{eV})$ & $\hbar \gamma(300 \mathrm{~K})(\mathrm{meV})$ & $\hbar \gamma(500 \mathrm{~K})(\mathrm{meV})$ \\
\hline $\mathrm{Au}$ & 9.0 & 35 & 58 \\
$\mathrm{Ti}$ & 2.51 & 47 & 78 \\
\hline \hline
\end{tabular}

Setting the relaxation parameter $\gamma$ in Eqs. (18) and (19) strictly to zero $[24,46]$, the dissipative Drude model is replaced by the dissipationless plasma model,

$$
\varepsilon_{p}(\omega)=1-\frac{\omega_{p}^{2}}{\omega^{2}}, \quad \varepsilon_{p}\left(i \xi_{l}^{(n)}\right)=1+\frac{\omega_{p}^{2}}{\xi_{l}^{(n)^{2}}} .
$$

An important difference between the plasma model and the Drude model consists of the fact that the Drude model is temperature dependent whereas the plasma model is not. Below, we perform numerical computations of the nonequilibrium Casimir pressure using both models. For the plate material, we choose either gold or titanium which possess rather different Drude parameters as listed in Table I based on Ref. [47].

Computations of the magnitude of the nonequilibrium Casimir pressure (1) are performed using Eqs. (3)-(7) and (9)-(15). While Eq. (3) is expressed in terms of a summation over the Matsubara frequencies, Eq. (9) is presented in terms of integrals of oscillating integrands over real frequencies. It is common knowledge that a problem of numerical convergence in the Lifshitz formula at real frequencies refers to only the total Casimir pressure and is unrelated to the thermal correction $[48,49]$ due to the presence of the factor $\Theta(\omega, T)$. In our case, Eq. (9) is very much like the thermal correction, and the convergence is ensured by a presence of the factors $\Theta\left(\omega, T_{m}\right)$.

The computational results as functions of the separation between the plates are shown in Fig. 2(a) for $\mathrm{Au}$ and in Fig. 2(b) for Ti. The top line in Fig. 2(a) is computed using the plasma model (20), and the bottom line is computed using the Drude model (18) and (19). Each of these lines is actually a superposition of two lines computed for plates of $d=20-\mathrm{nm}$ and $1-\mu \mathrm{m}$ thicknesses. Note that even the plates of minimum thickness considered $(d=20 \mathrm{~nm})$ allow theoretical description in terms of the bulk dielectric permittivity. For instance, according to results of Refs. [50,51], the Casimir pressure can be calculated using the bulk dielectric permittivity if the plates made of $\mathrm{Au}$ consist of more than 30 atomic layers, i.e., are of more than 7-nm thickness. Approximately the same results are expected for Ti plates.

The pairs of lines labeled 1 and 2 in Fig. 2(b) are computed for plates of $d=20-\mathrm{nm}$ and $1-\mu \mathrm{m}$ thicknesses, respectively. The upper line in each pair was obtained using the plasma model whereas the lower line corresponds to the Drude model. As can be seen in Fig. 2, the value of $P_{\text {neq }}$ essentially depends on the model employed for the dielectric permittivity. Although, for Au plates, there is basically no dependence on the plate thickness $d$ for the values chosen, the dependence is more pronounced for Ti plates. 

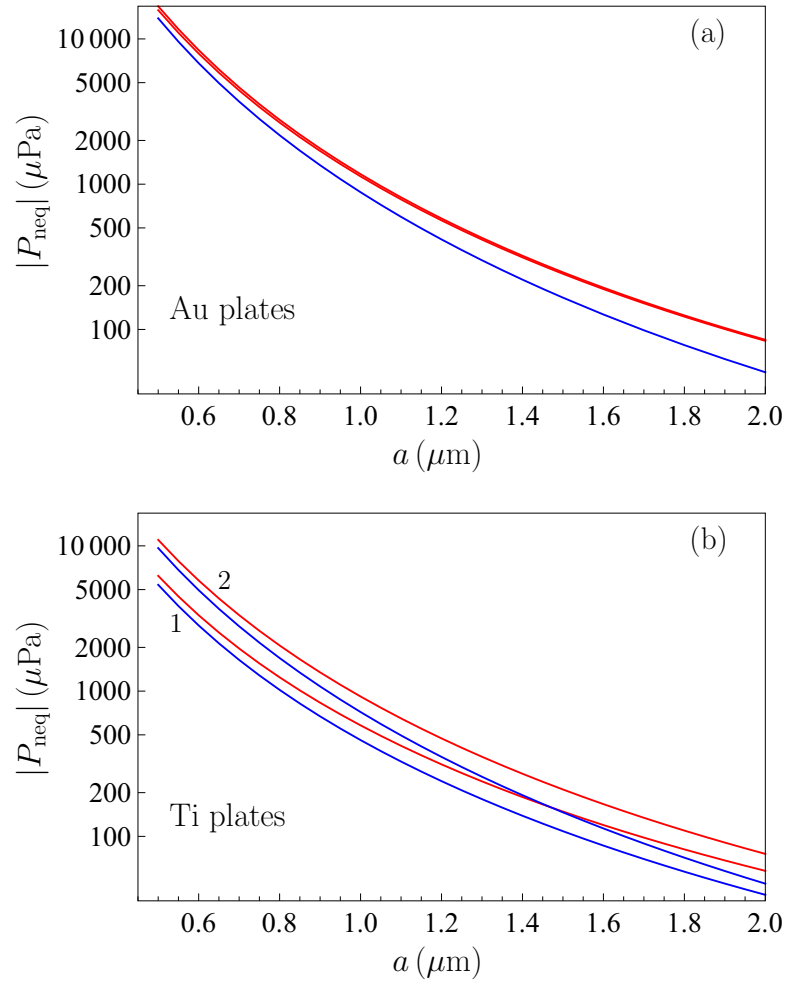

FIG. 2. The magnitudes of the nonequilibrium Casimir pressure are shown as functions of separation (a) for $\mathrm{Au}$ plates by the top and bottom lines computed using the plasma and Drude models, respectively, and (b) for Ti plates by the pairs of lines labeled 1 and 2 computed for the plates of $d=20-\mathrm{nm}$ and $1-\mu \mathrm{m}$ thicknesses, respectively, where, in each pair, the upper and lower lines are obtained using the plasma and Drude models, respectively.

To clearly demonstrate the difference in the values of $P_{\text {neq }}$ caused by the use of different permittivity models for plates of different thicknesses, we have also computed the quantity $P_{\text {neq }} / P_{0}$ where

$$
P_{0}(a)=-\frac{\pi^{2}}{240} \frac{\hbar c}{a^{4}}
$$

is the Casimir pressure between two ideal metal plates at zero temperature. The computational results are shown on a linear scale as function of the separation $a$ for Au in Fig. 3(a) and Ti in Fig. 3(b). In Fig. 3(a), the top and bottom pairs of the solid lines are computed using the plasma and Drude models, respectively. In each of these pairs, the lower solid line corresponds to $d=20 \mathrm{~nm}$, and the upper solid line corresponds to $d=1 \mu \mathrm{m}$. In Fig. 3(b), the pairs of solid lines labeled 1 and 2 are computed for the plates with $d=20-\mathrm{nm}$ and $1-\mu \mathrm{m}$ thicknesses, respectively. In each pair, the upper solid line is obtained using the plasma model, and the lower solid line is obtained using the Drude model. In both Figs. 3(a) and 3(b), the dashed lines are computed using the Drude model with the temperature-independent relaxation parameter fixed at its value at $300 \mathrm{~K}$.

As Fig. 3 shows, the theoretical predictions based on the Drude and plasma models differ more than sufficiently in order to discriminate between them experimentally. For
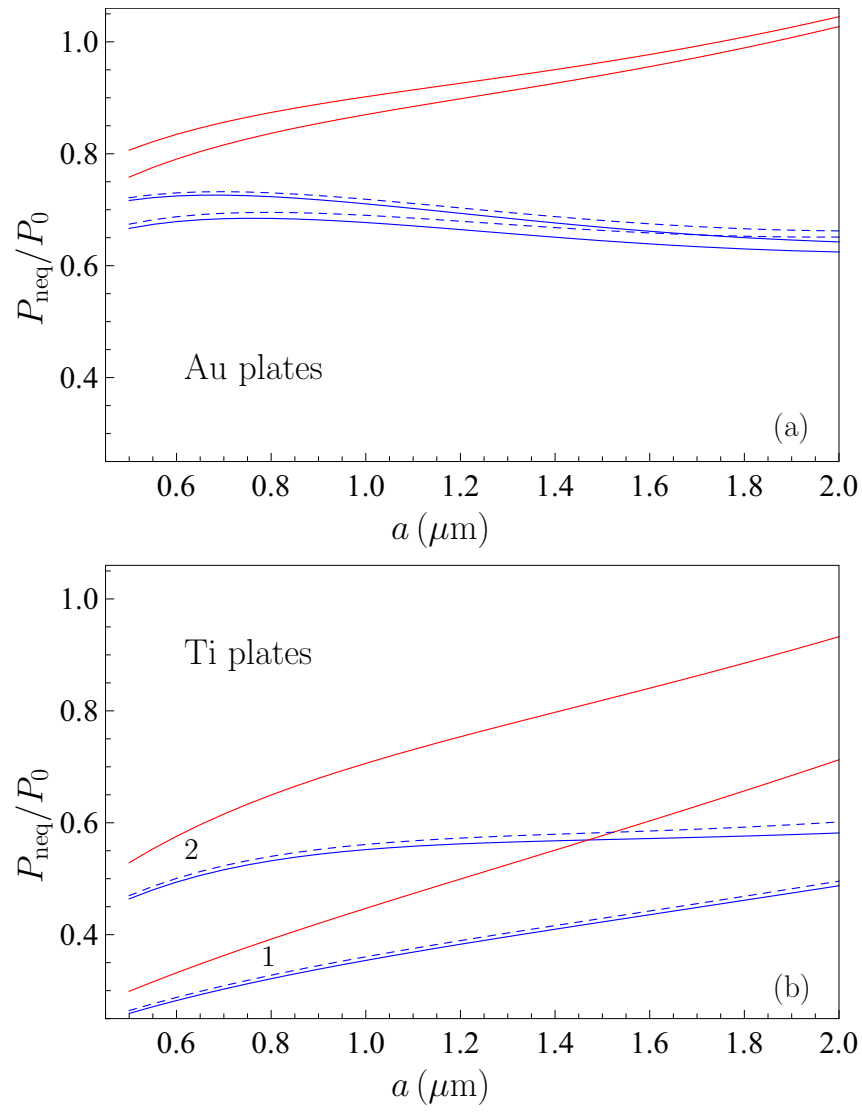

FIG. 3. The nonequilibrium Casimir pressures normalized to the Casimir pressure between two ideal metal plates at zero temperature are shown as functions of separation (a) for Au plates by the top and bottom pairs of solid lines computed using the plasma and Drude models, respectively, where, in each pair, the lower and upper solid lines are for plate thicknesses of $d=20 \mathrm{~nm}$ and $1 \mu \mathrm{m}$, respectively, and (b) for Ti plates by the pairs of solid lines labeled 1 and 2 computed for the plate thicknesses of $d=20 \mathrm{~nm}$ and $1 \mu \mathrm{m}$, respectively, where, in each pair, the upper and lower solid lines are obtained using the plasma and Drude models, respectively. In all cases, the dashed lines are computed by means of the Drude model with the temperature-independent relaxation parameter taken at $300 \mathrm{~K}$.

instance, for $\mathrm{Au}$ plates separated by $a=0.5,1$, and $2 \mu \mathrm{m}$, the relative differences between the nonequilibrium Casimir pressures computed using the Drude and plasma models are equal to $12 \%, 22 \%$, and $39 \%$ for plates of 20 -nm thickness, respectively. For plates of $1-\mu \mathrm{m}$ thickness, the respective relative differences are $11 \%, 21 \%$, and $38 \%$, i.e., the dependence on the thickness is very minor. For Ti plates, approximately the same results are obtained. Furthermore, from Fig. 3, one can again see that the thickness makes a greater impact on $P_{\text {neq }}$ for Ti plates than for Au plates. The differences between the dashed lines and the neighboring solid lines illustrate the effect originating from the temperature dependence of the dielectric permittivity. Below, we consider them in more detail.

According to Sec. II, a dependence of the dielectric permittivity on temperature makes an impact on both the equilibrium and the nonequilibrium contributions to the total 


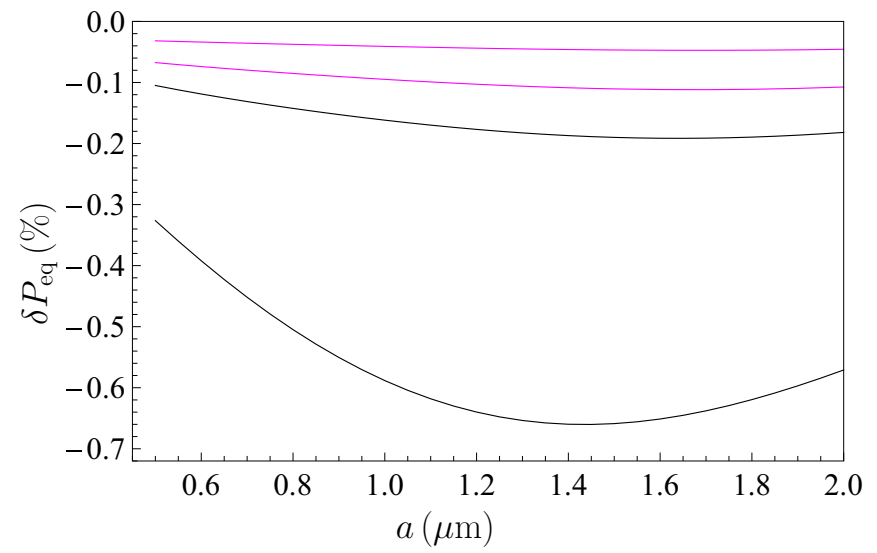

FIG. 4. The relative difference between the modified and the standard equilibrium contributions to the nonequilibrium Casimir pressure between two similar metallic plates described by the Drude model with the temperature-dependent relaxation parameter as a function of separation is shown in percentages by the four lines from top to bottom for Au plates with $d=1-\mu \mathrm{m}$ and 20-nm thicknesses and for Ti plates with $d=1 \mu \mathrm{m}$ and $20 \mathrm{~nm}$, respectively.

nonequilibrium Casimir pressure (1). We begin with the first of them and consider the relative difference,

$$
\delta P_{\mathrm{eq}}\left(a, T_{1}, T_{2}\right)=\frac{\widetilde{P}_{\mathrm{eq}}\left(a, T_{1}, T_{2}\right)-\bar{P}_{\mathrm{eq}}\left(a, T_{1}, T_{2}\right)}{\bar{P}_{\mathrm{eq}}\left(a, T_{1}, T_{2}\right)},
$$

between $\widetilde{P}_{\text {eq }}$ and $\bar{P}_{\text {eq }}$ defined in Eqs. (3) and (2), respectively, The computations are performed for the dielectric permittivity of the Drude model. For the plasma model, it is evident that $\delta P_{\text {eq }} \equiv 0$.

In Fig. 4, the computational results for $\delta P_{\text {eq }}$ in percentages are shown as functions of the plate separation by the four lines from top to bottom for the cases of Au plates with $d=1 \mu \mathrm{m}$ and $20 \mathrm{~nm}$ and Ti plates with $d=1 \mu \mathrm{m}$ and $20 \mathrm{~nm}$, respectively. As can be seen in Fig. 4, for Au plates, the magnitudes of the relative difference between $\widetilde{P}_{\text {eq }}$ and $\bar{P}_{\text {eq }}$ do not exceed $0.1 \%$ independently of the values of plate thickness and separation distance. For Ti plates, the impact of the plate thickness on $\delta P_{\text {eq }}$ is more pronounced, but, even in this case, the magnitudes of $\delta P_{\text {eq }}$ at different separations do not reach $0.7 \%$ for plates of $20-\mathrm{nm}$ thickness. One can conclude that an impact of the temperature dependence of the dielectric permittivity of a metal on the equilibrium contribution to the nonequilibrium Casimir pressure, although of interest theoretically, is negligibly small from the experimental point of view.

Now, we consider the role of the second contribution to the nonequilibrium Casimir pressure (1). For similar plates, it arises only if the dielectric permittivity of the plate material is temperature dependent. In Fig. 5, the ratios $\Delta P_{\text {neq }} / P_{\text {neq }}$ are shown as functions of the plate separation by the two lines from top to bottom for (a) Au plates of $d=1-\mu \mathrm{m}$ and 20-nm thicknesses and (b) Ti plates of $d=20-\mathrm{nm}$ and 1- $\mu \mathrm{m}$ thicknesses. Computations are performed using the dielectric permittivity of the Drude model (18) and (19). For the plasma model, one has $\Delta P_{\text {neq }} \equiv 0$.
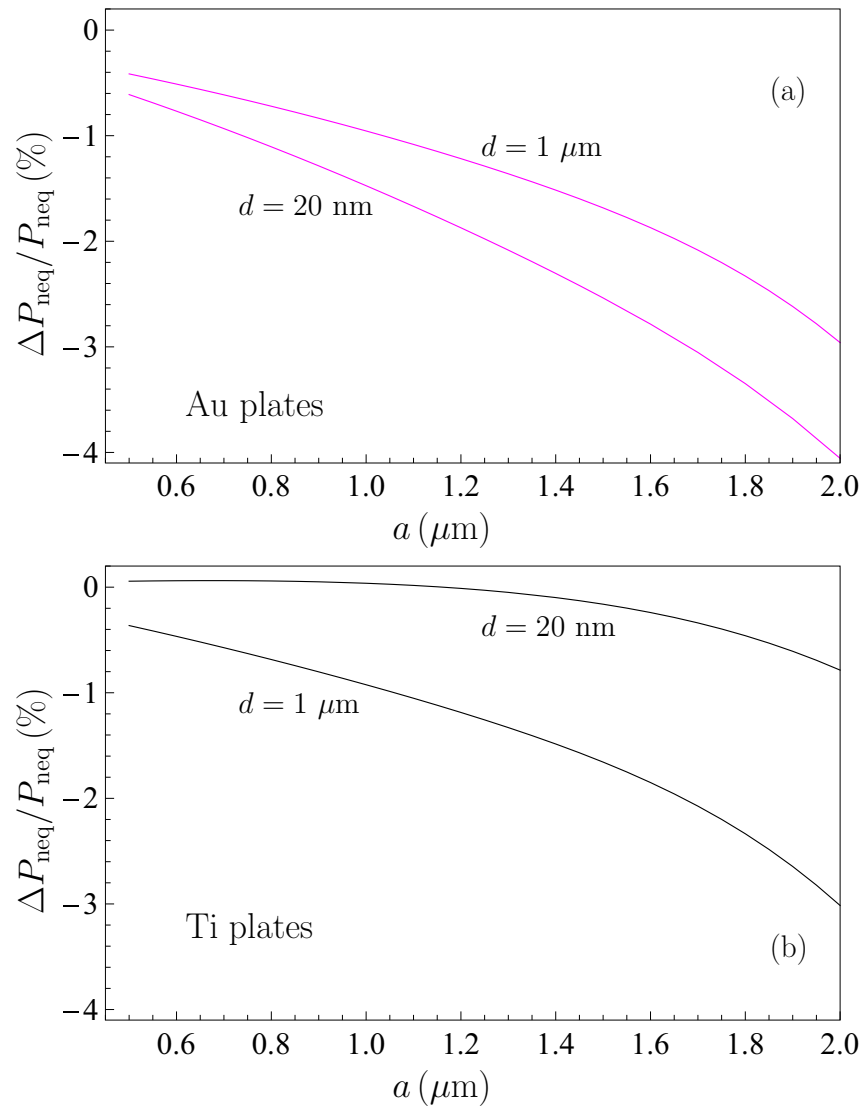

FIG. 5. The ratios of the proper nonequilibrium contribution and the total Casimir nonequilibrium pressure are shown in percentages as a function of the separation by the two solid lines from top to bottom for (a) Au plates with $d=1-\mu \mathrm{m}$ and 20-nm thicknesses, respectively, and (b) Ti plates with $d=20 \mathrm{~nm}$ and $1 \mu \mathrm{m}$, respectively.

As can be seen from Fig. 5(a), for Au plates of 20$\mathrm{nm}$ thickness, the proper nonequilibrium contribution $\Delta P_{\text {neq }}$ reaches $4 \%$ of the magnitude of the total nonequilibrium Casimir pressure at a separation of $a=2 \mu \mathrm{m}$ between the plates. Although $P_{\text {neq }}$ is negative, $\Delta P_{\text {neq }}$ is positive, i.e., it makes a repulsive contribution over the range of separations considered. For Ti plates of 1- $\mu \mathrm{m}$ thickness, $\Delta P_{\text {neq }}$ reaches $3 \%$ of $\left|P_{\text {neq }}\right|$ at $a=2 \mu \mathrm{m}$. It should be noted that, for Ti plates of 20-nm thickness at less than $1.19-\mu \mathrm{m}$ separation, $\Delta P_{\text {neq }}$ changes its sign and becomes negative, i.e., it has the same sign as $P_{\text {neq. Thus, at sufficiently small separations between }}$ the plates, it contributes to the standard Casimir attraction.

Physically, the results are explained by the difference in penetration depth $c / \omega_{p}$ for electromagnetic fluctuations in $\mathrm{Au}$ and $\mathrm{Ti}$ of 22 and $78.9 \mathrm{~nm}$, respectively. Although, for $\mathrm{Au}$, the thinnest plate considered is almost of the same thickness as the penetration depth, for Ti the penetration depth exceeds the thickness of the thinnest plate considered by almost a factor of 4 .

We can conclude that, for similar plates of sufficient thickness and with temperature-dependent dielectric permittivity, the proper nonequilibrium contribution $\Delta P_{\text {neq }}$ can amount to several percent of the total pressure. It should, thus, be taken into account when comparing experiment and theory. 


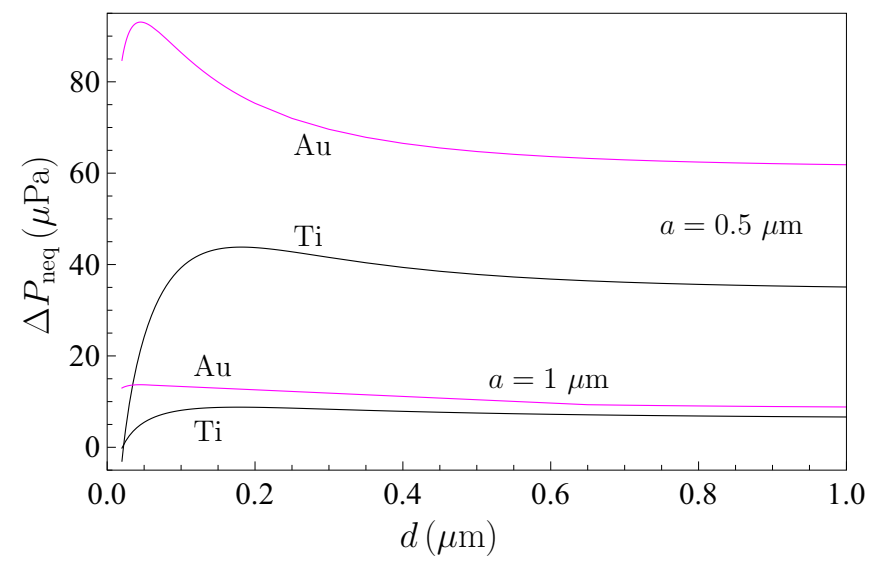

FIG. 6. The proper nonequilibrium contribution to the Casimir pressure is shown as a function of plate thickness by the top and bottom pairs of lines computed at separations $a=0.5$ and $1 \mu \mathrm{m}$ between the plates, respectively. In each pair, the upper line corresponds to Au plates whereas the lower line is for Ti plates.

For completeness, we have also calculated the proper nonequilibrium contribution to the Casimir pressure $\Delta P_{\text {neq }}$ as a function of plate thickness $d$. The computational results are presented in Fig. 6 by the top and bottom pairs of lines obtained at separations between the plates $a=0.5$ and $1 \mu \mathrm{m}$, respectively. The upper line in each pair is plotted for $\mathrm{Au}$ plates, and the lower line is plotted for Ti plates. As can be seen in Fig. 6, the quantity $\Delta P_{\text {neq }}$ reaches its maximum value for plate thicknesses $d$ approximately equal to twice the penetration depth of electromagnetic fluctuations in $\mathrm{Au}$ and Ti. For $\mathrm{Au}$, the quantity $\Delta P_{\text {neq }}$ remains positive for any plate thickness in the range from $20 \mathrm{~nm}$ to $1 \mu \mathrm{m}$, i.e., corresponds to a repulsive contribution to the Casimir force.

From Fig. 6, it can be seen that, for Ti plates of thickness $d \geqslant 22.1 \mathrm{~nm}$ at a distance of $a=0.5 \mu \mathrm{m}, \Delta P_{\text {neq }}$ is also positive. For thinner Ti plates, however, $\Delta P_{\text {neq }}$ changes sign and becomes negative. For instance, for Ti plates of thickness $d=$ $20 \mathrm{~nm}$, we have $\Delta P_{\text {neq }}=-3.04 \mu \mathrm{Pa}$. For Ti plates spaced at $a=1 \mu \mathrm{m}$, we have $\Delta P_{\text {neq }}>0$ for plates of thickness $d \geqslant 21.1 \mathrm{~nm}$. For thinner plates, $\Delta P_{\text {neq }}<0$. Specifically, for plates with $d=20 \mathrm{~nm}$, one finds $\Delta P_{\text {neq }}=-0.17 \mu \mathrm{Pa}$. We underline that, for similar Ti plates of some specific value of the plate thickness depending on the plate distance, the proper nonequilibrium contribution to the Casimir pressure vanishes. In this case, the total nonequilibrium pressure (1) is reduced to the equilibrium contribution $\widetilde{P}_{\text {eq }}$ alone.

At the end of this section, we recall that all the above results are formulated for the total pressure $P_{\text {neq }}$ on the lower plate. According to Eq. (17), the total pressure on the upper plate $\widehat{P}_{\text {neq }}$ is obtained by an addition of the constant contribution. For the temperatures used in our computations $\left(T_{1}=300\right.$ and $T_{2}=500 \mathrm{~K}$ ), this contribution amounts to

$$
\frac{2 \sigma}{3 c}\left(T_{2}^{4}-T_{1}^{4}\right)=6.86 \mu \mathrm{Pa} .
$$

From a comparison with Fig. 6, it is seen that the constant term is of approximately the same size as $\Delta P_{\text {neq }}$ for plates at a separation of $a=1 \mu \mathrm{m}$.

\section{CONCLUSIONS AND DISCUSSION}

In this paper, we have applied the theory of Casimir forces out of thermal equilibrium to the case of two similar plates possessing a temperature-dependent dielectric permittivity. This problem, not studied so far, is of importance in connection with the so-called Casimir puzzle awaiting its resolution for already $20 \mathrm{yr}$. It is relevant also for developing applications of the Casimir force in various microdevices.

We suggested here that, if two similar plates possessing a temperature-dependent dielectric permittivity are kept at different temperatures, they can be considered as dissimilar bodies with different dielectric permittivities. This approach allows the application of a theory developed earlier in the literature [8,9]. According to our results and in contrast to what was believed so far for two identical bodies, the nonequilibrium Casimir pressure for two similar metallic plates possessing a temperature-dependent dielectric permittivity does not reduce to an equilibrium contribution alone but contains a nonzero proper nonequilibrium contribution as well. Furthermore, in this case, the equilibrium contribution undergoes a modification and is not equal to the mean of two standard equilibrium pressures calculated for pairs of plates kept at two different temperatures. The configuration of two dissimilar plates possessing a temperature-dependent dielectric permittivity can be treated in an analogous way.

The developed formalism was applied to metallic plates of equal finite thickness made of either $\mathrm{Au}$ or $\mathrm{Ti}$ and spaced at sufficiently large separations so that an influence of core electrons on the Casimir force is negligibly small. We have computed the total nonequilibrium Casimir pressure and both the equilibrium and the proper nonequilibrium contributions to it at different separations and plate thicknesses using the dielectric permittivities of the temperature-dependent Drude model and the temperature-independent plasma model. It is shown that the relative difference between the total nonequilibrium pressures computed using the Drude and plasma models reaches $39 \%$ when the separation distance between the plates varies from 0.5 to $2 \mu \mathrm{m}$. The magnitude of the relative difference between the modified and the standard equilibrium contributions to the total pressure is shown to be less than $1 \%$. However, according to our results, the proper nonequilibrium contribution to the total pressure between two similar plates computed using the Drude model can reach $4 \%$ of the magnitude of the total pressure for $\mathrm{Au}$ plates, i.e., it should be taken into account in the comparison of experiment and theory. It is interesting that, for Ti plates of a certain thickness depending on separation (as an example, for plates separated by $0.5 \mu \mathrm{m}$, this thickness equals $22.1 \mathrm{~nm}$ ), the proper nonequilibrium contribution to the total pressure vanishes and changes sign to yield an attractive contribution for thinner plates.

To conclude, according to the above results, the nonequilibrium Casimir pressure between similar plates made of a material possessing a temperature-dependent dielectric permittivity demonstrates not only quantitative, but also qualitative effects, which are lacking for bodies described by a temperature-independent permittivity and may be of interest for future experiments and technological applications. These 
effects could be observed using the experimental setup described in Refs. [43,52] where the upper plate plays the role of a sensor suspended by the springs and the lower plate is mounted on a piezoelectric stage. Taking into account that an immediate application of the above computational results requires the vacuum conditions outside of both plates, their thicknesses should be chosen sufficiently large (typically more than $0.5 \mu \mathrm{m}$ ) in order that a material underlying the lower plate will not impact on the nonequilibrium pressure. One could also suggest other types of experimental setups suitable for observation of the proposed effects.

For the future, it is planned to explore situations where the effects described in this paper are more pronounced, e.g., for materials for which the dielectric permittivity is more sensitive to changes in temperature. The most interesting candidate for such a study is graphene, leading to a large thermal effect in the Casimir interaction at short separations $[53,54]$.

\section{ACKNOWLEDGMENTS}

The work of G.L.K. and V.M.M. was partially supported by Peter the Great Saint Petersburg Polytechnic University in the framework of the Program "5-100-2020." V.M.M. was partially funded by the Russian Foundation for Basic Research, Grant No. 19-02-00453 A. His work was also partially supported by the Russian Government Program of Competitive Growth of Kazan Federal University. G.L.K. and V.M.M. are grateful to the Department of Physics of the University of Augsburg where part of this work was performed for kind hospitality. They acknowledge helpful discussions with M. Antezza and V. B. Svetovoy.
[1] R. H. French, V. A. Parsegian, R. Podgornik, R. F. Rajter, A. Jagota, J. Luo, D. Asthagiri, M. K. Chaudhury, Y.-m. Chiang, S. Geanick et al., Long range interactions in nanoscale science, Rev. Mod. Phys. 82, 1887 (2010).

[2] E. M. Lifshitz, The Theory of Molecular Attractive Forces between Solids, Zh. Eksp. Teor. Fiz. 29, 94 (1955) [Sov. Phys. JETP 2, 73 (1956)].

[3] I. E. Dzyaloshinskii, E. M. Lifshitz, and L. P. Pitaevskii, General theory of van der Waals forces, Usp. Fiz. Nauk 73, 381 (1961) [Sov. Phys. Usp. 4, 153 (1961)].

[4] I. A. Dorofeyev, The force of attraction between two solids with different temperatures, J. Phys. A 31, 4369 (1998).

[5] C. Henkel, K. Joulain, J.-P. Mulet, and J.-J. Greffet, Radiation forces on small particles in thermal near fields, J. Opt. A: Pure Appl. Opt. 4, S109 (2002).

[6] M. Antezza, L. P. Pitaevskii, and S. Stringari, New Asymptotic Behavior of the Surface-Atom Force out of Thermal Equilibrium, Phys. Rev. Lett. 95, 113202 (2005).

[7] G. Bimonte, A Theory of Electromagnetic Fluctuations for Metallic Surfaces and van der Waals Interactions between Metallic Bodies, Phys. Rev. Lett. 96, 160401 (2006).

[8] M. Antezza, L. P. Pitaevskii, S. Stringari, and V. B. Svetovoy, Casimir-Lifshitz Force Out of Thermal Equilibrium and Asymptotic Nonadditivity, Phys. Rev. Lett. 97, 223203 (2006).

[9] M. Antezza, L. P. Pitaevskii, S. Stringari, and V. B. Svetovoy, Casimir-Lifshitz force out of thermal equilibrium, Phys. Rev. A 77, 022901 (2008).

[10] G. Bimonte, Scattering approach to Casimir forces and radiative heat transfer for nanostructured surfaces out of thermal equilibrium, Phys. Rev. A 80, 042102 (2009).

[11] R. Messina and M. Antezza, Scattering-matrix approach to Casimir-Lifshitz force and heat transfer out of thermal equilibrium between arbitrary bodies, Phys. Rev. A 84, 042102 (2011).

[12] R. Messina and M. Antezza, Casimir-Lifshitz force out of thermal equilibrium and heat transfer between arbitrary bodies, Europhys. Lett. 95, 61002 (2011).

[13] M. Krüger, T. Emig, and M. Kardar, Nonequilibrium Electromagnetic Fluctuations: Heat Transfer and Interactions, Phys. Rev. Lett. 106, 210404 (2011).
[14] M. Krüger, T. Emig, G. Bimonte, and M. Kardar, Non-equilibrium Casimir forces: Spheres and sphere-plate, Europhys. Lett. 95, 21002 (2011).

[15] M. Krüger, G. Bimonte, T. Emig, and M. Kardar, Trace formulas for nonequilibrium Casimir interactions, heat radiation, and heat transfer for arbitrary bodies, Phys. Rev. B 86, 115423 (2012).

[16] R. Messina and M. Antezza, Three-body radiative heat transfer and Casimir-Lifshitz force out of thermal equilibrium for arbitrary bodies, Phys. Rev. A 89, 052104 (2014).

[17] A. Noto, R. Messina, B. Guizal, and M. Antezza, CasimirLifshitz force out of thermal equilibrium between dielectric gratings, Phys. Rev. A 90, 022120 (2014).

[18] G. Bimonte, T. Emig, M. Krüger, and M. Kardar, Dilution and resonance-enhanced repulsion in nonequilibrium fluctuation forces, Phys. Rev. A 84, 042503 (2011).

[19] V. B. Bezerra, G. Bimonte, G. L. Klimchitskaya, V. M. Mostepanenko, and C. Romero, Thermal correction to the Casimir force, radiative heat transfer, and an experiment, Eur. Phys. J. C 52, 701 (2007).

[20] A. I. Volokitin and B. N. J. Persson, Near-field radiative heat transfer and noncontact friction, Rev. Mod. Phys. 79, 1291 (2007).

[21] A. I. Volokitin and B. N. J. Persson, Theory of the interaction forces and the radiative heat transfer between moving bodies, Phys. Rev. B 78, 155437 (2008).

[22] I. Latella, P. Ben-Abdallah, S.-A. Biehs, M. Antezza, and R. Messina, Radiative heat transfer and nonequilibrium CasimirLifshitz force in many-body systems with planar geometry, Phys. Rev. B 95, 205404 (2017).

[23] F. Tajik, Z. Babamahdi, M. Sedighi, A. A. Masoudi, and G. Palasantzas, Dependence of non-equilibrium Casimir forces on material optical properties toward chaotic motion during device actuation, Chaos 29, 093126 (2019).

[24] G. L. Klimchitskaya, U. Mohideen, and V. M. Mostepanenko, The Casimir force between real materials: Experiment and theory, Rev. Mod. Phys. 81, 1827 (2009).

[25] L. M. Woods, D. A. R. Dalvit, A. Tkatchenko, P. RodriguezLopez, A. W. Rodriguez, and R. Podgornik, Materials 
perspective on Casimir and van der Waals interactions, Rev. Mod. Phys. 88, 045003 (2016).

[26] M. Bordag, G. L. Klimchitskaya, U. Mohideen, and V. M. Mostepanenko, Advances in the Casimir Effect (Oxford University Press, Oxford, 2015).

[27] R. S. Decca, E. Fischbach, G. L. Klimchitskaya, D. E. Krause, D. López, and V. M. Mostepanenko, Improved tests of extradimensional physics and thermal quantum field theory from new Casimir force measurements, Phys. Rev. D 68, 116003 (2003).

[28] R. S. Decca, D. López, E. Fischbach, G. L. Klimchitskaya, D. E. Krause, and V. M. Mostepanenko, Precise comparison of theory and new experiment for the Casimir force leads to stronger constraints on thermal quantum effects and long-range interactions, Ann. Phys. (NY) 318, 37 (2005).

[29] R. S. Decca, D. López, E. Fischbach, G. L. Klimchitskaya, D. E. Krause, and V. M. Mostepanenko, Tests of new physics from precise measurements of the Casimir pressure between two gold-coated plates, Phys. Rev. D 75, 077101 (2007).

[30] R. S. Decca, D. López, E. Fischbach, G. L. Klimchitskaya, D. E. Krause, and V. M. Mostepanenko, Novel constraints on light elementary particles and extra-dimensional physics from the Casimir effect, Eur. Phys. J. C 51, 963 (2007).

[31] C.-C. Chang, A. A. Banishev, R. Castillo-Garza, G. L. Klimchitskaya, V. M. Mostepanenko, and U. Mohideen, Gradient of the Casimir force between Au surfaces of a sphere and a plate measured using an atomic force microscope in a frequency-shift technique, Phys. Rev. B 85, 165443 (2012).

[32] A. A. Banishev, C.-C. Chang, G. L. Klimchitskaya, V. M. Mostepanenko, and U. Mohideen, Measurement of the gradient of the Casimir force between a nonmagnetic gold sphere and a magnetic nickel plate, Phys. Rev. B 85, 195422 (2012).

[33] A. A. Banishev, G. L. Klimchitskaya, V. M. Mostepanenko, and U. Mohideen, Demonstration of the Casimir Force between Ferromagnetic Surfaces of a Ni-Coated Sphere and a Ni-Coated Plate, Phys. Rev. Lett. 110, 137401 (2013).

[34] A. A. Banishev, G. L. Klimchitskaya, V. M. Mostepanenko, and U. Mohideen, Casimir interaction between two magnetic metals in comparison with nonmagnetic test bodies, Phys. Rev. B 88, 155410 (2013).

[35] G. Bimonte, D. López, and R. S. Decca, Isoelectronic determination of the thermal Casimir force, Phys. Rev. B 93, 184434 (2016).

[36] J. Xu, G. L. Klimchitskaya, V. M. Mostepanenko, and U. Mohideen, Reducing detrimental electrostatic effects in Casimir-force measurements and Casimir-force-based microdevices, Phys. Rev. A 97, 032501 (2018).

[37] M. Liu, J. Xu, G. L. Klimchitskaya, V. M. Mostepanenko, and U. Mohideen, Examining the Casimir puzzle with an upgraded AFM-based technique and advanced surface cleaning, Phys. Rev. B 100, 081406(R) (2019).
[38] M. Liu, J. Xu, G. L. Klimchitskaya, V. M. Mostepanenko, and U. Mohideen, Precision measurements of the gradient of the Casimir force between ultraclean metallic surfaces at larger separations, Phys. Rev. A 100, 052511 (2019).

[39] M. Hartmann, G.-L. Ingold, and P. A. Maia Neto, Plasma versus Drude Modeling of the Casimir Force: Beyond the Proximity Force Approximation, Phys. Rev. Lett. 119, 043901 (2017).

[40] M. Hartmann, G.-L. Ingold, and P. A. Maia Neto, Advancing numerics for the Casimir effect to experimentally relevant aspect ratios, Phys. Scr. 93, 114003 (2018).

[41] G. L. Klimchitskaya and V. M. Mostepanenko, Experiment and theory in the Casimir effect, Contemp. Phys. 47, 131 (2006).

[42] G. Bimonte, Observing the Casimir-Lifshitz force out of thermal equilibrium, Phys. Rev. A 92, 032116 (2015).

[43] G. L. Klimchitskaya, V. M. Mostepanenko, and R. I. P. Sedmik, Casimir pressure between metallic plates out of thermal equilibrium: Proposed test for the relaxation properties of free electrons, Phys. Rev. A 100, 022511 (2019).

[44] M. Born and E. Wolf, Principles of Optics (Cambridge University Press, Cambridge, U.K., 1999).

[45] J. M. Obrecht, R. J. Wild, M. Antezza, L. P. Pitaevskii, S. Stringari, and E. A. Cornell, Measurement of the Temperature Dependence of the Casimir-Polder Force, Phys. Rev. Lett. 98, 063201 (2007).

[46] G.-L. Ingold, A. Lambrecht, and S. Reynaud, Quantum dissipative Brownian motion and the Casimir effect, Phys. Rev. E 80, 041113 (2009).

[47] M. A. Ordal, R. J. Bell, R. W. Alexander, L. L. Long, and M. R. Querry, Optical properties of fourteen metals in the infrared and far infrared: Al, $\mathrm{Co}, \mathrm{Cu}, \mathrm{Au}, \mathrm{Fe}, \mathrm{Pb}, \mathrm{Mo}, \mathrm{Ni}, \mathrm{Pd}, \mathrm{Pt}, \mathrm{Ag}, \mathrm{Ti}, \mathrm{V}$, and W, Appl. Opt. 24, 4493 (1985).

[48] V. B. Svetovoy, Evanescent character of the repulsive thermal Casimir force, Phys. Rev. A 76, 062102 (2007).

[49] G. Bimonte, G. L. Klimchitskaya, and V. M. Mostepanenko, Comment on "Temperature dependence of the Casimir force for lossy bulk media”, Phys. Rev. A 84, 036501 (2011).

[50] M. Boström, C. Persson, and B. E. Sernelius, Casimir force between atomically thin gold films, Eur. Phys. J. B 86, 43 (2013).

[51] G. L. Klimchitskaya and V. M. Mostepanenko, Casimir and van der Waals energy of anisotropic atomically thin metallic films, Phys. Rev. B 92, 205410 (2015).

[52] G. L. Klimchitskaya, V. M. Mostepanenko, R. I. P. Sedmik, and $\mathrm{H}$. Abele, Prospects for searching thermal effects, nonnewtonian gravity and axion-like particles: CANNEX test of the quantum vacuum, Symmetry 11, 407 (2019).

[53] G. Gómez-Santos, Thermal van der Waals interaction between graphene layers, Phys. Rev. B 80, 245424 (2009).

[54] G. L. Klimchitskaya and V. M. Mostepanenko, Origin of large thermal effect in the Casimir interaction between two graphene sheets, Phys. Rev. B 91, 174501 (2015). 